\title{
ON GROUPS WHICH ACT FREELY ON VECTOR BUNDLES OVER SPHERES
}

\author{
F. E. A. JOHNSON
}

\begin{abstract}
Let $G \simeq K \times Q$ be the semidirect product of a finitely presented group $K$ of type FP and a finite group $Q$ of periodic cohomology. It is shown that $G$ acts freely and properly discontinuously on a vector bundle over a sphere.
\end{abstract}

Let $G$ be a finitely presented group of type VFP [5], and periodic Farrell cohomology. C.T.C. Wall has conjectured that $G$ acts freely and properly discontinuously on the product of a sphere and a Euclidean space with, in general, noncompact quotient. The simplest form of this group theoretic behaviour occurs when $G$ is a semidirect product $G \cong K \rtimes Q$ where $K$ is a finitely presented group of type FP and $Q$ is a finite group with periodic cohomology. In this note we show that such $G$ act freely and properly discontinuously on a vector bundle over a sphere. In retrospect this seems a more natural formulation of the above conjecture, though we are unable to dispose of the conjecture in Wall's original formulation. We begin with

THEOREM 1. Let $G \cong K \rtimes Q$ be the semidirect product of a finitely presented group $K$ of type $F L$ (as kernel) and a finite group $Q$ with periodic cohomology. Then $G$ acts freely and properly discontinuously on the total space of a smooth fibre bundle over a sphere with fibre a Euclidean space.

Proof. Write $G$ as the middle term in an exact sequence thus: $\mathcal{E}=(K$ $\rightarrow G \rightarrow Q$ ) and let $B \mathcal{E}$ be the geometrical realisation of the associated minimal Kan fibration, $B \mathcal{E}=(B K \rightarrow B G \rightarrow B Q) . B E$ is locally trivial in the category of compactly generated spaces [2]. By the main result of Swan's thesis [8], we may choose a finite simplicial complex $Y_{Q}$ with fundamental group $Q$ and universal covering $\tilde{Y}_{Q}$ homotopy equivalent to a $k$-sphere $S^{k}$, for some $k$. Let $X_{Q}$ be an open regular neighbourhood of $Y_{Q}$ in some high dimensional Euclidean space $\mathbf{R}^{m+k}$. Since $S^{k}$ and $\tilde{Y}_{Q}$ have the same simple homotopy type, then $\tilde{X}_{Q}$ is also an open regular neighbourhood for $S^{k}$ provided $m$ is sufficiently large [9]. Hence we may assume that $\tilde{X}_{Q}$ is diffeomorphic to $\mathbf{R}^{m} \times S^{k}$.

By hypothesis on $K$ we may choose a finite complex $Y_{K}$ homotopy

Received by the editors April 7, 1978.

AMS (MOS) subject classifications (1970). Primary 57E30; Secondary 2000.

Key words and phrases. Group of type VFP, periodic Farrell cohomology, fibre smoothing theorem, free proper discontinuous action. 
equivalent to $B K$. Let $X_{K}$ be an open regular neighbourhood of $Y_{K}$ in $\mathbf{R}^{n}$ for large enough $n(n>2(m+k)$ at least). By taking a further product with $\mathbf{R}$ if necessary, we can suppose that $\tilde{X}_{K}$ is diffeomorphic to $\mathbf{R}^{n}$ as in [6], [4], so we assume this as well.

Let $c: X_{Q} \rightarrow B Q$ induce an isomorphism of fundamental groups, and put $\xi=c^{*}(B \mathscr{E})=\left(B K \rightarrow E_{\xi} \rightarrow X_{Q}\right) . \xi$ is locally trivial, since $X_{Q}$ is locally compact, hence is a Hurewicz fibration. We now apply the open fibre smoothing theorem of Casson and Gottlieb (Theorem A of [1]) to deduce that $\xi$ is fibre homotopy equivalent to a smooth fibre bundle $\eta=\left(X_{K} \rightarrow X_{G} \rightarrow\right.$ $X_{Q}$ ) with fibre $X_{K}$ and base $X_{Q}$. If $\hat{X}_{G}$ denotes the covering of $X_{G}$ with $\pi_{1}\left(\hat{X}_{G}\right)=K$, then $\hat{X}_{G}$ is a smooth bundle over $\tilde{X}_{Q}=\mathbf{R}^{m} \times S^{k}$ with fibre $X_{K}$, hence the universal cover $\tilde{X}_{G}$ fibres over $\mathbf{R}^{m} \times S^{k}$ with fibre $\mathbf{R}^{n} \simeq \tilde{X}_{K}$. By projecting onto $S^{k}$ and using the contractibility of $\mathbf{R}^{m}$, we see that $\tilde{X}_{G}$ fibres smoothly over $S^{k}$ with fibre $\mathbf{R}^{m+n}$. The result follows since $G$ acts freely and properly discontinuously on $\tilde{X}_{G}$. Q.E.D.

To get from smooth $\mathbf{R}^{N}$ bundles to vector bundles, we can use the following piece of folklore, which is by no means the strongest possible statement.

Proposition 2. Let $E$ be the total space of a smooth $\mathbf{R}^{N}$ bundle over a smooth closed manifold $M^{k}$. Suppose that (a) $k+N>5$ and (b) $N>$ $\max (k, 2)$. Then $E$ is diffeomorphic to the total space of a vector bundle over $M$.

Proof. Since $\mathbf{R}^{N}$ is contractible, we may construct a section $i: M \rightarrow E$ for $\xi$, which, since $2 \operatorname{dim} M<\operatorname{dim} E$, we may suppose is an immersion. Hence $i(M)$ is a smooth submanifold of $E$. Let $N_{1}, N_{2}$ be smooth closed tubular neighbourhoods of $i(M)$ with $N_{1} \subset$ Int $N_{2}$. By uniqueness of tubular neighbourhoods, we see that Int $N_{2}-$ Int $N_{1} \cong \partial N_{1} \times[0,1)$. Since $N>3$, then $E-$ Int $N_{1}$ is an open $h$-cobordism. Since $k+N \geqslant 5$, we may apply the open $h$-cobordism theorem of Stallings [7] to conclude that $E-\operatorname{Int} N_{1}$ $\cong \partial N_{1} \times[0,1)$. Consequently, $E$ is diffeomorphic to Int $N_{2}$, which is the total space of a normal vector bundle. Q.E.D.

Putting together Theorem 1 and Proposition 2 we obtain

THEOREM 3. Let $G \cong K \rtimes Q$ be the semidirect product of a finitely presented group of type FP and a finite group $Q$ with periodic cohomology. Then $G$ acts freely and properly discontinuously on the total space of a vector bundle over a sphere.

Proof. If $K$ is of type FL, the result follows from Theorem 1 and Proposition 2. It is easily checked that the construction of Theorem 1, provided $k \geqslant 1$ and $m \geqslant 1$, automatically satisfies the subsidiary conditions of Proposition 2. Now, it is unknown whether every finitely presented group of type FP is also of type FL. If $K$ is not of type FL, then $K \times \mathbf{Z}$ is of type FL, by Gersten's product formula for Wall's finiteness obstruction [3]. Then 
$G \times \mathbf{Z} \cong(K \times \mathbf{Z}) \rtimes Q$, and since $G \times \mathbf{Z}$ acts freely and properly discontinuously on a vector bundle over a sphere, so does $G$. Q.E.D.

\section{REFERENCES}

1. A. Casson and D. H. Gottlieb, Fibrations with compact fibres, Amer. J. Math. 99 (1977), 159-189.

2. P. Gabriel and M. Zisman, Calculus of fractions and homotopy theory, Ergebnisse der Math. Wissenschaften, Springer-Verlag, Berlin, 1967.

3. S. M. Gersten, A product formula for Wall's obstruction, Amer. J. Math. 83 (1966), 337-346.

4. F. E. A. Johnson, Manifolds of homotopy type $K(\pi, 1)$. I, Proc. Cambridge Philos. Soc. 70 (1971), 387-393.

5. J. P. Serre, Cohomologie des groupes discrets, Prospects in Mathematics, Ann. of Math. Studies, no. 70, Princeton University Press, Princeton, N.J., 1971, pp. 77-169.

6. J. R. Stallings, The piecewise-linear structure of Euclidean space, Proc. Cambridge Philos. Soc. 58 (1962), 481-487.

7. On finite processes leading to differentiability in the complement of a point, Differential and Combinatorial Topology, Princeton University Press, Princeton, N.J., 1965.

8. R. G. Swan, Periodic resolutions for finite groups, Ann. of Math. (2) 72 (1960), 267-291.

9. J. H. C. Whitehead, Simplicial spaces, nuclei, and m-groups, Proc. London Math. Soc. 45 (1939), 243-337.

Department of Mathematics, University Collbge London, Gower Strket, London, WC1E 6BT, UNTRD KINGDOM 